\title{
A Survey of Selected Neurological Signs in Schizophrenia Research
}

Emanuel H. Rosen, M.D.

Menninger Clinic, Topeka, Kansas

Follow this and additional works at: https://jdc.jefferson.edu/jeffjpsychiatry

Part of the Psychiatry Commons

Let us know how access to this document benefits you

\section{Recommended Citation}

Rosen, M.D., Emanuel H. (1991) "A Survey of Selected Neurological Signs in Schizophrenia Research," Jefferson Journal of Psychiatry. Vol. 9 : Iss. 1 , Article 6.

DOI: https://doi.org/10.29046/JJP.009.1.004

Available at: https://jdc.jefferson.edu/jeffjpsychiatry/vol9/iss $1 / 6$

This Article is brought to you for free and open access by the Jefferson Digital Commons. The Jefferson Digital Commons is a service of Thomas Jefferson University's Center for Teaching and Learning (CTL). The Commons is a showcase for Jefferson books and journals, peer-reviewed scholarly publications, unique historical collections from the University archives, and teaching tools. The Jefferson Digital Commons allows researchers and interested readers anywhere in the world to learn about and keep up to date with Jefferson scholarship. This article has been accepted for inclusion in Jefferson Journal of Psychiatry by an authorized administrator of the Jefferson Digital Commons. For more information, please contact: JeffersonDigitalCommons@jefferson.edu. 


\title{
A Survey of Selected Neurological Signs in Schizophrenia Research
}

\author{
Emanuel H. Rosen, M.D.
}

\begin{abstract}
This review covers three areas of schizophrenia research not usually addressed in residents' curricula: smooth pursuit eye movements and genetics, orienting responses, and tardive dyskinesia. The significance of these neurological signs is explored in terms of their clinical symptom associations and their heuristic value. Neuroanatomical aspects of these signs is noted to be speculatively consistent with hypofrontality and negative symptoms in schizophrenia. The author describes the nature of occulomotor abnormalities and discusses an applicable research study. He summarizes relevant areas of genetic research including linkage analysis and marker studies and explains some of the orienting response abnormalities in schizophrenia. He then examines tardive dyskinesia for associated clinical psychological implications beyond its manifestation as a movement disorder. Finally, the author discusses a study of eye movement abnormalities in patients with tardive dyskinesia to illustrate how different research perspectives can be interwoven to tie genetically inherited neurological signs to the schizophrenic illness.
\end{abstract}

\section{INTRODUCTION}

This paper is a selective review of some areas of schizophrenia research which are not routinely addressed in resident curricula. The neurological deficits noted in Smooth Pursuit Eye Movements (SPEM), Orienting Responses, and Tardive Dyskinesia (TD) are often associated with alterations in schizophrenic symptoms and contain important clues as to the nature of what schizophrenic patients experience. The possible neuroanatomical localizations of these signs often fit with data from newer imaging techniques implicating frontal lobe dysfunction in schizophrenia. The significance of tardive dyskinesia as sometimes being a possible sign of a particular symptomatic subtype of schizophrenia is reviewed in some detail. The overarching theme of this paper is that these various neurophysiological deficits have their clinical correlates as well as heuristic value in thinking about nosological and neuroanatomical issues in schizophrenia research.

\section{SPEM and Genetics}

SPEM research dates back to 1910. When "normal" people are asked to track a slow moving target, such as a child on a swing, their eyes do so in a smooth movement, that is, uninterrupted by jerking catch-up movements or saccades. Schizophrenic 
people, however, do not follow targets with such grace. A subgroup of them (40\% to $80 \%$ ) substitute saccades of varying sizes and forms for smooth pursuit (1). This phenomenon is so robust that it has been replicated in every published study since 1974 (2).

SPEM abnormalities provide an objective, valid, and reliable abnormality that may aid in the highly subjective art and science of psychiatric diagnosis. For example, schizotypal personality disorder patients, when compared to normal people and to people with other types of personality disorders, have an increased incidence of impaired eye tracking similar to that found in schizophrenic individuals. This finding is consistent with the diagnostic conceptualization of schizotypal personality disorder as part of a biologically based thought disorder spectrum (3).

The term "gain" in eye tracking research refers to the ratio of the speed of the eyeball to the speed of the target. The percent RMS (root-mean-square) error is simply a global measure of overall pursuit performance. Normals and schizophrenics both have a decrease in pursuit gain and an increase in percent RMS error when they are asked to track a randomly alternating and hence unpredictable target as opposed to a steadily oscillating predictable one. The unpredictability results in slower tracking and more mistakes in tracking as the individual cannot anticipate and eventually learn the target's path. However, the type of mistakes that the schizophrenic makes in tracking an unpredictable target is different than those made by normals (4).

Normals err in making both "catch-up" and "look-ahead" (anticipatory) saccades while schizophrenics make many "catch-up" but relatively few "look-ahead" saccades. In addition, normals have higher pursuit gain for both predictable and unpredictable targets. The lack of anticipation, and diminished velocity to a lesser extent, meshes nicely with speculations of frontal lobe impairment from other areas of schizophrenia research, namely imaging studies. The diagram in Figure 1 highlights some of the neuroanatomy of smooth pursuits. The pivotal control of the frontal eye fields is depicted. Early studies of cerebral blood flow in normal individuals have shown that there is "hyperfrontality" or increased blood flow to anterior frontal (premotor and prefrontal) regions relative to posterior frontal regions. Schizophrenics were shown to have "hypofrontality" or a decreased antero-posterior flow gradient. Preliminary PET findings are consistent with decreased frontal lobe physiological functioning in schizophrenia as reflected in decreased glucose utilization. It is interesting that one study has demonstrated a relative lack of blood flow through the dorso-lateral prefrontal cortex when schizophrenic patients were asked to perform a task that normally increases flow to this area (5-6). The late Smadar Levin, who specialized in eye tracking research, theorized that such areas contiguous to frontal centers of ocular control were likely implicated in schizophrenia. As she deduced, this localization is consistent with other features of the illness as well: "Finally and most importantly, the clinical frontal lobe syndrome is the single organic brain syndrome that is most similar to the clinical syndrome of schizophrenia, particularly of chronic 'negative' symptoms schizophrenia (7).

While tardive dyskinesia will be discussed later in this paper, it is useful at this 
juncture to point out that many believe that all voluntary motor activity is regulated by the prefrontal cortex and the basal ganglia in conjunction with input from the frontal motor cortex. Perhaps this is why some tardive dyskinetic schizophrenics, who may have more negative symptoms and caudate damage than other schizophrenics, also have deficits in voluntary motor movements (8).

Genetics research has also benefited from the discovery of the pursuit anomaly. That this trait is inherited is demonstrated by the fact that $45 \%$ of the first-degree relatives of schizophrenic individuals manifest impaired tracking, in contrast to only $8 \%$ to $12 \%$ of normal individuals $(7,9)$. The significance of genetically transmitted traits is best appreciated in light of some understanding of the concepts of genetic markers and linkage analysis.

A genetic marker is a stable, relatively unique, observable manifestation that is transmitted from generation to generation together with an illness. A marker may be something totally unrelated to the illness, for example, red-green color blindness (10). The term marker has also been applied to denote a distinctive segment of DNA that may be inherited with the mutant disease gene at a higher frequency than other segments of the DNA (11). These two definitions are intimately related, the main difference being a focus on the gene versus its observable expression. The closer the physical proximity of the two genes on a chromosome, the less likely it is that they will be separated during miotic recombination, and hence in their transmission among family members. When two genes do separate, they are said to recombine. Thus the lower the percentage of time that genes recombine, the closer the genes are on the chromosome-that is, the more closely linked.

Linkage analysis provides clues to the chromosomal location of a defective gene. Linkage data is recorded by what is known as a lod score. A lod score is a logarithm of a statistical probability. Its calculation can be used as an indicator of likelihood that a marker and a disease are linked. A lod score of $>+3$ is considered highly indicative of linkage while one of $<-2$ indicates that the traits are almost certainly not linked (11-12).

There is a connection between linkage analysis and psychophysiological markers. Markers aid in identifying which members of a given family are most likely to have inherited the disease gene, even when they are clinically asymptomatic. A subpopulation of carriers or people at risk is then defined that can be studied intensively with the new technology. More precisely, their genes can be cleaved and analyzed. There is also an underlying assumption that biologically defined disease phenotypes will correlate better with underlying genetic makeup, which allows for a genetically more homogeneous disease population to be investigated (13). Finally, psychophysiological markers can help elucidate the mode of transmission of the disease gene within the family tree. Further research may vastly expand the scope and accuracy of genetic counseling and may make possible the desperately needed prospective investigations of people at high risk $(10,14)$.

The natural obstacles to be overcome by research include incomplete gene penetrance, variable expressivity (the tendency of the same gene defect to manifest itself in different ways), and genetic heterogeneity (the same exact disease state 
caused by either different mutations at the same gene site or by multiple mutations at many gene sites). Ideally, researchers try to find one bad gene causing one disease in one family; in reality they need very large families and serendipity to facilitate this complex search (12). Discovering the gene or genes that cause schizophrenia is also only the first hurdle, after which comes the daunting task of discovering how the given defect expression manifests itself in the human brain (15).

Chromosome 5 has been the focus of study at a number of research centers (16). A recently published case study told of a proband and his maternal uncle, both of whom had schizophrenia as well as the rare anomaly of a partial trisomy of chromosome 5. Only the proband and his uncle had abnormal SPEM; the normal family members had normal tracking. This clustering of anomalies suggests that the trisomy contributed to the illness (17). A current major hypothesis that eye movement dysfunction (EMD) and schizophrenia are alternative expressions of the same latent disease trait would explain why these conditions can occur together as well as separately (18).

In contrast to efforts to find the genetic causes of schizophrenia on a particular chromosome, some theoretical constructs propose a less reductionistic pattern of inheritance. One such exposition postulates that schizophrenia results from the complex interaction that includes not only innate genetic traits, environmental effects, and random phenomena, but also includes a disturbance in the balance between these factors and the so-called normative regulation of the background genotype. In other words, apart from the emphasis placed on finding a disease gene, this theory holds that, in fact, the background genes are more likely to play a definitive role in modifying the expression of genes that may or may not be present in normal individuals.

A similar model has proven useful in understanding cancer where it is thought that the regulator genes-not just the oncogenes-play a crucial role in determining who will actually develop cancer. The background genotype, in this theory, acts as a homeostatic stabilizing influence, like a restraining net, on genetic and environmental biases. If the net is broken, increased variability can be expected. In summary, this theory posits a model for understanding the interactions between genetic biases, the environment, and the background genotype. More specifically, it shows how they balance each other's influence in determining the behavior ultimately manifested by an organism (19).

\section{Electrodermal Orienting Responses}

Psychophysiology involves the study of the physiological responses associated with psychological states. One of the more consistent anomalous psychophysiological responses of schizophrenic patients is called skin conductance orienting response (SCOR), nonresponsiveness. The ability of the skin to conduct electricity is slightly enhanced by sweat, and this small increase in the skin conductance of an electric current can be measured by electrodes (20-21). An orienting response is the organism's first reaction to a new stimulus; it is associated with increased sweating 
and therefore with increased skin conductance. If a student is relaxing during the third hour of a preoedipal rage theory lecture and someone nearby drops a pen, then the student's body undergoes a physiological response, within approximately $150 \mathrm{~ms}$, to the new stimulus. To determine "what's up," the body orients its attentional abilities toward the sound of the pen hitting the floor. This phenomenon has been called "the most elementary form of attention," and it is the reaction that engages the complex brain attentional apparatus to assess the novel stimulus (22).

SCOR nonresponsiveness in schizophrenic individuals is intuitively not surprising given that, since Kraeplin, attention deficiency has been noted as a hallmark of schizophrenia (22). SCOR nonresponsiveness in a large subgroup of schizophrenic patients has been confirmed over and over again in American and multinational studies and has been considered "almost universally replicable." (22) Neuroleptic medications do not seem to affect responding status although this issue has not been conclusively resolved (23-24).

The clinical meaning of nonresponding is controversial and is now under active investigation along several lines of inquiry. First is the question of which clinical symptoms correlate with this phenomenon. In some studies, nonresponders seem to be more withdrawn and conceptually disorganized (20). However, a recent attempt to differentiate patients with positive and negative symptoms by responding status demonstrated instead that both positive and negative symptoms may be increased in nonresponders (24).

Hoping to advance understanding of the nature of the deficits in schizophrenia, scientists are trying to determine at what level the abnormality in attention processing lies. These patients appear to lack the ability to weigh which stimulus input is worthy of more processing, as opposed to possessing an inability to detect or filter out (22). While there were initially some reports that seemed to relate nonresponding to frontal lobe dysfunction, more current thinking is that the phenomenon still defies neuroanatomical localization (25). One PET scan pilot study, however, has found electrodermal nonresponsive schizophrenics have decreased metabolic activity in medial superior frontal gyri relative to responders (26).

It should be noted also that electrodermal variables-including the orienting response-are being applied to studies of high-risk subjects for their possible prognostic value. Studies of the genetic inheritance of these variables may elucidate their role as genetic traits associated with vulnerability to schizophrenia (20).

Recently, orienting responses were measured in a sample of 101 15-year-old boys. Intriguingly a lack of an orienting response was found to be significantly more prevalent among those boys who, 10 years later, had acquired a criminal record. Thus, electrodermal abnormalities may not be specific to schizophrenia (27).

\section{Tardive Dyskinesia}

Most psychiatry residents are intimately familiar with TD as an involuntary movement disorder that they observe and record frequently in their clinical work. Less widely recognized is TD's significance well beyond its effect on body movement. 
Clinicians generally take for granted that TD is associated with the prolonged use of neuroleptics. Ideally, however, a prospective study of unmedicated schizophrenic patients should be designed to observe the frequency at which they develop TD. This theoretical problem is not a trivial issue. Some evidence indicates that there is a baseline incidence of orofacial dyskinetic movement. This evidence mostly, although not exclusively, comes from preneuroleptic era observations; nevertheless, Waddington and Crow (28) recently reviewed this dormant body of literature and, despite its major methodological flaws, they found a strikingly uniform prevalence of choreoathetoid movement in schizophrenic populations not exposed to neuroleptics. Specifically, prevalence rates varied narrowly between $5.1 \%$ and $7.9 \%$ in all five studies, regardless of whether they were conducted in 1936 or 1971. Kraeplin and Bleuler, in the early 1900 s well before the advent of neuroleptics, described involuntary choreoathetoid movements in schizophrenic persons. Astonishingly, the possible clinical relevance of this phenomenon was noted as early as 1857 by Griesinger, who wrote how "persistent automatic grimacing" and "chorea like movements" were indicative of a poor prognosis in "adult lunatics." (29)

Perhaps consistent with this poor prognosis, TD may represent an exacerbation by neuroleptics of preexisting brain damage in vulnerable patients (30). A subgroup of these patients may have developed TD as part of their "schizophrenia," even without neuroleptic exposure. This possibility provides an additional reason for carefully examining how patients who develop TD differ clinically from those who do not; the results may shed some light on the brain pathology involved in their schizophrenia.

Studies have shown that patients with TD have more extensive deficits in a wide variety of areas than other patients. They are, indeed, sicker.

TD patients have worsened smooth pursuit eye movement. The deficit takes the peculiar form of large nontracking saccades. These patients seem to have a diminished ability to attend to the target, then try to catch up by using these large, quick eye movements (1). In a similar vein, some early evidence indicates that TD is associated with significantly slowed reaction time (31).

A prospective study demonstrated that patients who develop TD perform more poorly on psychometric testing (e.g., the WAIS) and have more soft neurological signs than do non-TD patients (32). A recent preliminary study also indicated that not only is there a direct relationship between TD symptom severity and cognitive deficit, as measured by the WAIS and Wechsler Memory Scales, but also that orofacial TD seems to have a stronger correlation than extremity or truncal TD (33). Another interesting preliminary study contrasted TD patients with Parkinson's patients; on the total scale of the Brief Psychiatric Rating Scale (BPRS), tardive dyskinetic patients had significantly higher ratings of psychopathology (34). Another piece of the jigsaw puzzle-although in a far corner-is the significantly higher degree of major affective disorder in the family history of patients with tardive dyskinesia $(32,34)$

In a recent review (30), 8 of 11 studies showed greater deterioration in dyskinetic 
patients in such areas as conceptual level, memory, orientation, and paired learning. Some studies also seemed to indicate an association between abnormal involuntary movement and negative symptoms, as well as associations with certain CT and pneumoencephalographic abnormalities such as atrophy and the ventricle-to-brain ratio. Recent MRI data indicate that dyskinetic patients may have significantly smaller caudate volumes although no significant differences were found in other brain areas (35). Although all these areas require further study, the special deficits (other than involuntary movement) manifested by TD patients are worth keeping in mind clinically, if only to determine whether such observations correspond with one's own impressions.

The Latin root tard means late, hence tardive dyskinesia has come to be defined as those involuntary choreoathetoid movements induced by neuroleptics that usually appear only after several months (at least 6) of treatment. From a research methodology point of view, these movements have been classified in several ways. In fact, it is partly because of the wide variety of classifications that facile generalizations from the data are of ten difficult to deduce. As with most phenomena in science, the closer we look, the more complicated simple things become, and the more subclassifications are needed.

A nice illustration of this tendency is a study that examined noradrenergic characteristics of dyskinetic patients (36). Research diagnostic criteria (RDC) recognize the fact that TD status fluctuates. To correctly ascertain the appropriate category, of a myriad of possibilities, for a patient's disorder (e.g., transient, withdrawal, persistent, masked), patients must be assessed at least twice (37). However, RDG classifications did not indicate significant differences in noradrenergic functioning among the 19 dyskinetic patients. It was only by creating a subclassification of patients with withdrawal-exacerbated TD (a non-RDC criteria subclassification) that significant plasma MHPG level differences were revealed. This distinction hints at a major underlying challenge for TD research; determining the relationship between the neuroleptic responsiveness of the movement disorder and the nature of the disorder itself. In brief, those patients whose movement disorder is sensitive to neuroleptics may have a different movement disorder than those patients whose movement disorder is unaffected by neuroleptics. In some unique neurochemical or structural way, the latter group's dyskinesia may be part of their schizophrenia, just as in Huntington's chorea the movement disorder is part of the same process causing the thought disorder (38).

"Increased Saccadic Distractibility in Tardive Dyskinesia: Functional Evidence for Subcortical GABA Dysfunction," (39) is a recent study that will be examined in some detail here to illustrate how the various neurological signs can weave a more full understanding of clinical and neuroanatomical schizophrenic phenomena. Using two paradigms, the researchers studied three patient groups: (1) 18 chronic schizophrenic patients with TD, (2) 16 schizophrenic patients without TD, and (3) 8 normal controls. In the first paradigm, the subject was told to look at a center target light, then toward a second target light when it was turned on. In the second paradigm (an 
antisaccade paradigm), the subject was asked to look in the opposite direction from the second light when it was turned on.

Two scores were obtained: latency in milliseconds and a distractibility score. The latency score was the time it took for the patient's eyes to start to move. The distractibility score simply was the number of times, in the second paradigm, that the patient mistakenly looked toward rather than away from the distracting light. For purposes of data analysis, the latency scores were divided into two categories: reflexive and volitional. Reflexive latencies were those made in the first paradigm (without distraction) and those made to the wrong side in the second paradigm (when the patient mistakenly looked toward the distracting light instead of away from it). Volitional latency concerned those saccades made consciously and correctly away from the distracting stimulus as had been requested and practiced. These responses were termed volitional because the patients successfully willed their eyes to avoid reflexively looking at the distraction.

Schizophrenic patients and normal individuals did not differ significantly in their reflexive saccadic latency. Each of them took the same amount of time to process and effect motorically reflexive movement. However, schizophrenic patients, regardless of their TD status, took significantly longer than controls to make correct, volitional saccades. Moreover, all schizophrenic patients made more errors on the second paradigm than the controls did. But among the schizophrenic patients, those with tardive dyskinesia had distractibility scores twice as high as non-TD patients. These results were replicated in a follow-up study that also examined SPEM. The authors found no reported quantitative differences between dyskinetic and nondyskinetic schizophrenic patients in SPEM (40), although this finding is not in accord with that of another study (1).

Residents are probably wondering, "What does all this mean?" The study shows in an objective, quantifiable, verifiable way, how schizophrenic patients differ from normal people. Similarly, the findings differentiate patients with tardive dyskinesia from patients without tardive dyskinesia; the TD patients being more distractible. By understanding the mechanisms and meanings behind these differences, we can gain a better appreciation of both the brain lesions and the actual experiences of our patients.

Some aspects of this study shed light on the nature of brain lesions. As Figure 1 illustrates, the control of saccadic eye movements is complex. In fact, in addition to the connections depicted, posterior parietal regions of the cortex are also involved. The reflexive saccades are probably mediated by areas of the superior colliculus and below in the brain stem. Because both schizophrenic and normal individuals had unimpaired reflexive saccades, it is less likely that these lower areas are affected in schizophrenia. However, the study did find that volitional saccades took longer for schizophrenic patients than for normal people. The literature implicating frontal and posterior parietal regions in the etiology of schizophrenia is voluminous, as is the literature implicating subcortical areas of the brain. Damage to these central processing areas could account for the delayed reactions of schizophrenic patients, 


\section{Neuroanatomy of Saccadic Eye Movements}

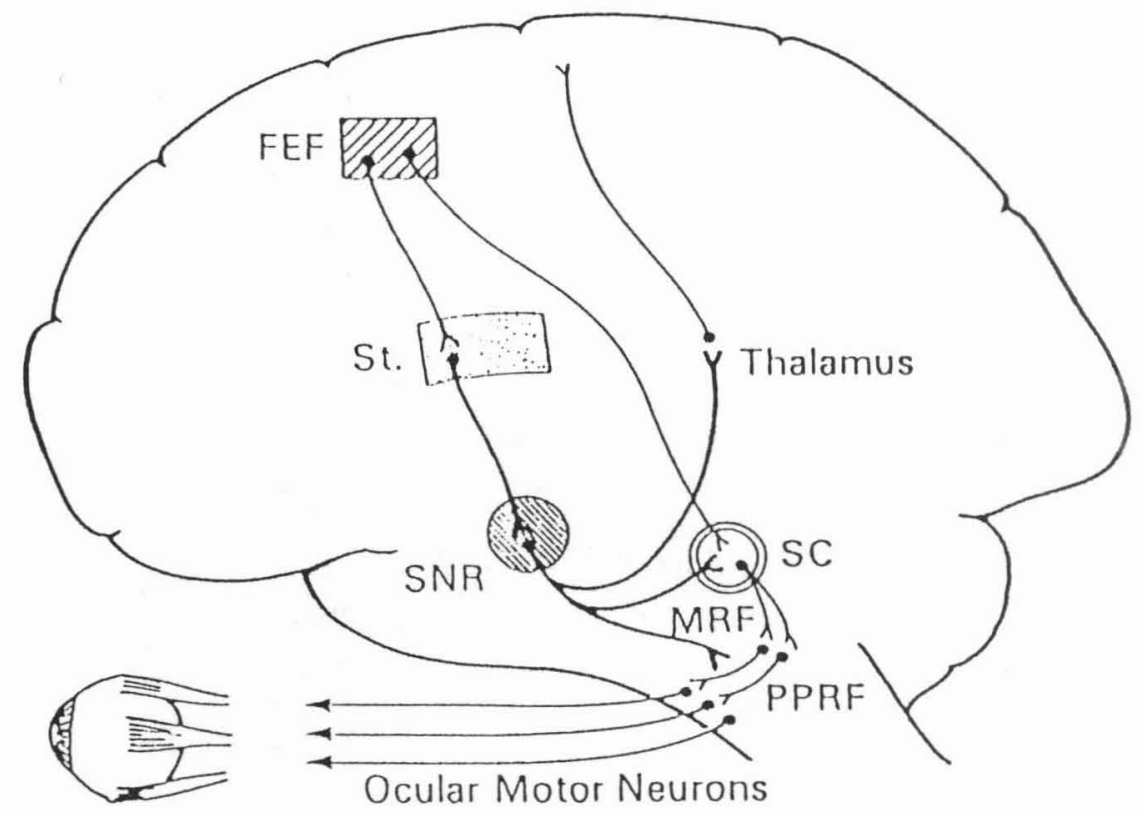

FEF - Frontal Eye Fields

St - Striatum

SNR - Substantia Nigra Pars Reticulata

SC - Superior Colliculus

MRF - Mesencephalic Reticular Formation

PPRF - Paramedian Pontine Reticular Formation

FIGURE 1. Neuroanatomy of saccadic eye movements. FEF, frontal eye fields; ST, striatum; SNR, substantia nigra pars reticulata; SC, superior colliculus; MRF, mesencephalic reticular formation; PPRF, paramedian pontine reticular formation.

and it could also account for a second finding-that schizophrenics showed more distractibility than controls.

As to why tardive dyskinetic schizophrenic patients showed more distractibility than nondyskinetic schizophrenic patients, the researchers posited a disruption of a specific pathway known as the nigrotectile pathway. Neuroanatomically, this pathway connects the substania nigra reticulata to the superior colliculus (see the diagram). Support of this neuroanatomical localization comes from animal studies in which a 
transection of this pathway in monkeys results in saccadic distractibility. A brief summary of the presumed neurochemistry of tardive dyskinesia in general may help to explain why this GABAnergic pathway is the hypothesized area of damage in TD patients.

Theories about the neurochemical basis of TD have evolved from schemes that consider only one neurotransmitter system-namely dopamine-to those that postulate complex interactions among dopamine, GABA, norepinephrine, and serotonin pathways. Classically, the neuroleptic's well-established blockage of dopamine at the synapse was thought to result in the "chemical denervation supersensitivity" of dopamine receptors, which, when stimulated, evoked involuntary movement (41). Inconsistencies in the experimental evidence from animal, human postmortem, and therapeutic trials have proven this scheme reductionistic. For example, since all patients quickly develop denervation supersensitivity from neuroleptics, why do only a fraction develop TD? Why the time lag with the side effect sometimes surfacing only after lengthy exposure over time to neuroleptic drugs (41-42)? Clearly, more complex explanations are required.

Speculation about norepinephrine's role in TD has led to trials of propranolol hydrochloride and clonidine hydrochloride (36) for its control. In this study, the influence of GABA dysregulation is highlighted. Early evidence indicates that GABA in the SNR is decreased in dyskinetic, but not in nondyskinetic, neurolepticized monkeys. The SNR to SC pathway is a major efferent GABA pathway linking the basal ganglia to eye movement. Cutting this pathway in monkeys results in saccadic distractibility that is perhaps analogous to the distractibility noted in human patients. It remains to be seen whether animal models will be correlated with human experience, but eye-movement study makes its contribution in this context. In brief, this study demonstrates a defect in the human functioning of the GABAnergic pathway that is present selectively in patients with tardive dyskinesia. Clinical trials of GABA agonists to treat TD are continuing, with some promising early results (41-44).

Some researchers believe that in the same way denervation supersensitivity of striatal dopaminergic regions may, in some cases, result in TD, neuroleptic denervation of mesolimbic or other nonstriatal regions may result in a supersensitivity psychosis. In this theoretical subgroup, withdrawal of neuroleptics results in a sudden worsening of psychosis (and possibly dyskinesia as well) with rapid improvement if medication is restored. These patients may display "tolerance" to neuroleptics (i.e., they require progressively higher doses to maintain the same therapeutic effect). In brief, a pernicious and persistent change in the patient's disease is induced (45-47).

\section{CONCLUSION}

The research literature indicates that patients who suffer from the movement disorder tardive dyskinesia tend also to suffer from more severe deficits in their psychological experiences (48). This phenomenon may be reflected clinically by a predominance in these patients of negative symptoms and cognitive dysfunction 
$(30,8,49-52)$. Efforts to understand schizophrenia as a neuropsychological entity can be enhanced by appreciating the ties between the neurological signs discussed and the clinical syndrome. Localizing the schizophrenic's lesions with newer imaging techniques has often implicated frontal lobe dysfunction, a region possibly implicated by these signs as well. Knowledge of the biological evidence supporting the clinical picture should enhance resident clinicians' understanding of the nature of their patients' afflictions.

\section{REFERENCES}

1. Spohn HE, Coyne L, Spray J: The effect of neuroleptics and tardive dyskinesia on smooth-pursuit eye movement in chronic schizophrenics. Arch Gen Psychiatry 45:833-840, 1988

2. Holzman PS: Recent studies of psychophysiology in schizophrenia. Schizophr Bulletin 13(1):49-75, 1987

3. Siever LJ, Keefe R, Bernstein DP: Eye tracking impairment in clinically identified patients with schizotypal personality disorder. Am J Psychiatry 147(6):740-745, 1990

4. Allen JS, Matsunaga K, Hacisalihzade S, Stark L: Smooth pursuit eye movements of normal and schizophrenic subjects tracking an unpredictable target. Biol Psychiatry 28:705-720, 1990

5. Devous MD Sr: Imaging brain function by single-photon emission computer tomography. In Andreasen NC (Ed.), Brain imaging: applications in psychiatry. Washington DC, American Psychiatric Press, 1989, pp 147-234

6. Berman KF, Weinberger DR: Schizophrenia: brain structure and function. In Kaplan HI, Sadock BJ (Eds.), Comprehensive textbook of psychiatry/V. Baltimore, Williams and Wilkins, pp 705-717, 1989

7. Levin S: Frontal lobe dysfunctions in schizophrenia-I. eye movement impairments. J Psychiat Res 18(1):27-55, 1984

8. Manschreck TC, Keuthen NJ, Schneyer ML, Celada MT, Laughery J, Collins P: Abnormal involuntary movements and chronic schizophrenic disorders. Biol Psychiatry 27:150-158, 1990

9. Spohn HE, Strauss ME: Relation of neuroleptic and anticholinergic medication to cognitive functions in schizophrenia. J Abnormal Psycho 98(4):367-380, 1989

10. Iacono WG: Psychophysiology and genetics: a key to psychopathology research. Psychophysiology 20(4):371-383, 1983

11. White R, Lalouel JM: Chromosome mapping with DNA markers. Sci Am 258(2):40-48, 1988

12. Breakefield XO, Bressman, S: Molecular genetics of movement disorders in movement disorders 2. Edited by Marsden CD, Fahn S. London, Butterworth, pp 33-44, 1987

13. Pato CN, Lander ES, Schulz SC: Prospects for the genetic analysis of schizophrenia. Schizophr Bull 15(3):365-372, 1989

14. Mullen MJ, Murray RM: The impact of molecular genetics on our understanding of the psychoses. Br J Psychiatry 154:591-595, 1988

15. Schulz SC, Pato CN: Advances in the genetics of schizophrenia: editor's introduction. Schizophr Bull 15(3):361-363, 1989

16. Gurling HM, Sherrington RP, Brynjolfsson J, et al.: Recent and future molecular genetic research into schizophrenia. Schizophr Bull 15(3):373-382, 1989 
17. Iacono WG, Basset AS, Jones BD: Eye tracking dysfunction is associated with partial trisomy of chromosome 5 and schizophrenia [letter to the editor]. Arch Gen Psychiatry 45:1140-1141, 1988

18. Holzman PS: The use of eye movement dysfunctions in exploring the genetic transmission of schizophrenia. Eur Arch Psychiatry Neurol Sci 239(1):43-48, 1989

19. Kovach JK: Genetic regulation and the pathogenesis of schizophrenia: conceptual and heuristic considerations. Behavioral and Brain Sciences, in press, 1991

20. Dawson ME, Nuechterlein KH: Psychophysiological dysfunctions in the developmental course of schizophrenic disorders. Schizophr Bull 10(2):204-232, 1984

21. Green S: Physiological psychology: an introduction. London, Routledge \& Kegan Paul, 1987

22. Bernstein AS: Orienting response research in schizophrenia: where we have come and where we might go. Schizophr Bull 13:623-640, 1987

23. Spohn HE, Coyne L, Wilson JK, Hayes K: Skin-conductance orienting response in chronic schizophrenics: the role of neuroleptics. J Abnorm Psychol 98:478-486, 1989

24. Green MF, Nuechterlein KH, Satz P: The relationship of symptomatology and medication to electrodermal activity in schizophrenia. Psychophysiology 26:148-157, 1989

25. Bernstein A: Personal communication, March 21, 1991

26. Hazlett EA, Dawson ME, Buchsbaum MF, Nuechterlein KH: Reduced regional brain glucose metabolism by PET in electrodermal nonresponder schizophrenics: a pilot study. Paper presented at the meeting of the Society for Psychophysiological Research in Boston, October 1990

27. Raine A, Phil D, Venables PH, Williams M: Autonomic orienting response in 15-year-old male subjects and criminal behavior at age 24. Am J Psychiatry 147:933-937, 1990

28. Waddington JL, Crow TJ: Abnormal involuntary movements and psychosis in the preneuroleptic era and in unmedicated patients: implications for the concept of tardive dyskinesia. In Wolf ME, Mosnaim AD (Eds.), Tardive dyskinesia: biological mechanisms and clinical aspects. Washington DC, American Psychiatric Press, pp 49-66, 1988

29. Awouters F, Niemegeers CJ, Janssen PA: "Tardive" dyskinesia: etiological and therapeutic aspects. Pharmacopsychiatry 23:33-37, 1990

30. Waddington JL: Tardive dyskinesia in schizophrenia and other disorders: associations with ageing, cognitive dysfunction and structural brain pathology in relation to neuroleptic exposure. Human Psychopharmacology: Clinical and Experimental 2:11-22, 1987

31. Spohn HE, Coyne L, Lacoursiere R, Mazur D, Hayes K: Relation of neuroleptic dose and tardive dyskinesia to attention, information-processing, and psychophysiology in mediated schizophrenics. Arch Gen Psychiatry 42:849-859, 1985

32. Wegner JT, Catalano F, Gibralter, J, Kane JM: Schizophrenics with tardive dyskinesia: neuropsychological deficit and family psychopathology. Arch Gen Psychiatry 42:860-865, 1985

33. DeWolfe AS, Ryan JJ, Wolf ME: Cognitive sequelae of tardive dyskinesia. J Nerv Ment Dis, 176:270-274, 1988

34. Richardson MA, Pass R, Bregman Z: Contrasts between patients' characteristics associated with tardive dyskinesia versus those associated with parkinsonism-like symptoms. In Wolf ME, Mosnaim AD (Eds.), Tardive dyskinesia: biological mechanisms and clinical aspects. Washington DC, American Psychiatric Press, pp 107-122, 1988

35. Mion CC, Andreasen NC, Arndt S, Swayze VW, II, Cohen GA: MRI abnormalities in tardive dyskinesia, unpublished manuscript. 
36. Glazer WM: Noradrenergic function and tardive dyskinesia. Psychiatric Annals 19(65):297301,1989

37. Singh H, Simpson GM: Tardive dyskinesia: clinical features in tardive dyskinesia biological mechanism and clinical aspects. In Wolf ME, Mosnaim AD (Eds.), Washington DC, American Psychiatric Press, pp 67-86, 1988

38. Glazer WM, Morgenstern H: Predictors of occurrence, severity, and course of tardive dyskinesia in an outpatient population. J Clin Psychopharmacol 8(4S):10S-16S, 1988

39. Thaker GK, Nguyen JA, Tamminga CA: Increased saccadic distractibility in tardive dyskinesia: functional evidence for subcortical GABA dysfunction. Biol Psychiatry 25:49 59, 1989

40. Thaker GK, Nguyen JA, Tamminga CA: Saccadic distractibility in schizophrenic patients with tardive dyskinesia [letter to the editor]. Arch Gen Psychiatry 46:755-756, 1989

41. Lieberman J: Dopamine pathophysiology in tardive dyskinesia. Psychiatric Annals 19(6): 289-296, 1989

42. Smith RC: Is the dopaminergic supersensitivity theory of tardive dyskinesia valid? In Wolf ME, Mosnaim AD (Eds.), Tardive dyskinesia: biological mechanisms and clinical aspects. Washington DC, American Psychiatric Press, pp 1-22, 1988

43. Thaker GK, Ferraro TN, Hare T, Tamminga CA: Pathophysiology and therapy of tardive dyskinesia: the GABA connection. In Wolf MD, Mosnaim AD (Eds.), Tardive dyskinesia: biological mechanisms and clinical aspects. Washington DC, American Psychiatric Press, pp 197-216, 1988

44. Nguyen JA, Thaker GK, Tamminga CA: Gamma-aminobytyric acid (GABA) pathways in tardive dyskinesia. Psychiatric Annals 19(6):302-309, 1989

45. Chouinard G, Jones BD: Neuroleptic-induced supersensitivity psychosis: clinical and pharmacologic characteristics. Am J Psychiatry 137(1):16-21, 1980

46. Peet M: Supersensitivity psychosis [letter to the editor].J Clin Psychiatry 52(2):90, 1991

47. Simpson GM, Singh H, Hunt JI, Vitiello B: Reply to letter to the editor. J Clin Psychiatry 52(2):90, 1991

48. Spohn HE: Personal communication, November 15, 1990

49. Jeste DV, Karson CN, Iager A, Bigelow LB, Wyatt RJ: Association of abnormal involuntary movements and negative symptoms. Psychopharmacol Bull 20:380-381, 1984

50. Bartzokis G, Hill MA, Altshuler L, Cummings JL, Wirsching W, May PRA: Tardive dyskinesia in schizophrenic patients: correlation with negative symptoms. Psychiatry Res 28:145-151, 1989

51. McCreadie RG, Barron ET, Winslow GS: The Nithsdale Schizophrenia Survey: II. abnormal movements. Br J Psychiatry 140:587-590, 1982

52. Guy W, Ban TA, Wilson WH: An international survey of tardive dyskinesia. Prog Neuropsychopharmacol Biol Psychiatry 9:401-405, 1985 